\title{
Comparison of Neuronavigation and Frame-Based Stereotactic Systems in Implanting Epileptic Depth Electrodes
}

\author{
Zhi HOU ${ }^{1 *}$, Xin $\mathrm{CHEN}^{1 *}$, Xian-Jun SHI ${ }^{1}$, Ning AN¹, Mei-Hua YANG ${ }^{1}$, Hui YANG ${ }^{1}$, Dong ZHANG², Shi-Yong LIU1 \\ ${ }^{1}$ The Third Military Medical University, Xinqiao Hospital, Epilepsy Center of PLA, Department of Neurosurgery, Chongqing, China \\ ${ }^{2}$ The Third Military Medical University, Xinqiao Hospital, Department of Radiology, Chongqing, China
}

*Zhi HOU and Xin CHEN are the co-first authors.

\section{ABSTRACT}

AIM: To investigate the application of neuronavigation in the implantation of depth electrodes in patients with epilepsy.

MATERIAL and METHODS: Thirty-six patients with epilepsy who were implanted with depth electrodes using neuronavigation were assessed for accuracy of implantation and associated complications.

RESULTS: In the imaging navigation group, patients were implanted with 2-14 depth electrodes. The average number of implantations was 4.8 electrodes/case. The average implantation error was $2.03 \pm 0.98 \mathrm{~mm}$, exhibiting no significant difference compared to the frame-based stereotactic group. In the imaging group, an average of 19.4 min was required to implant each electrode, which was significantly shorter than the time required in the frame group ( $34.5 \mathrm{~min})$. The temporal lobe was elucidated as the factor that affects electrode implantation accuracy. One patient in the imaging group exhibited a small amount of bleeding, and one suffered from cerebrospinal fluid leakage; however, the overall complication rate in the imaging group was lower than that in the frame group.

CONCLUSION: Imaging navigation provides better means of depth electrode implantation; its implantation accuracy is similar to that of the frame-based stereotactic method and it is less time consuming and causes less complications, and is especially suitable for stereoelectroencephalography, which requires multiple depth electrodes.

KEYWORDS: Neuronavigation, Intracranial electrode implantation, Stereoelectroencephalography

\section{INTRODUCTION}

Intracranial electrode tracing has become an important means for evaluating the epileptogenic focus, especially in patients whose epileptogenic loci cannot be located using electroencephalography and other non-invasive assessment tools. Invasive intracranial electroencephalography (iEEG) is the preferred means of determining the exact locations of epileptogenic foci $(12,21)$. Intracranial electrodes can be classified as epidural, subdural cortical (including strip-like and grid-like electrode), and depth electrodes, of which the depth electrode has been widely used in epilepsy surgery, and plays an important role in the epileptogenic focus evaluation of the medial temporal lobe $(2,21)$.
Previously, depth electrode implantation was usually performed using the frame-based stereotactic method (14). The frame-based stereotactic system has high positioning precision, and is suitable for precise positioning and puncturing towards deep structures; for example in stereotactic biopsy or deep brain stimulation (DBS), which need accurate positioning $(3,6)$. The disadvantages of this method, however, are evident: 1) The puncturing process is complicated and time consuming, making it difficult to implant multiple electrodes (e.g. for stereoelectroencephalography [SEEG]) (16); 2) It is difficult to adjust the puncture direction and path in real-time, especially from a poor posture (16); 3) The puncturing process might injure blood vessels and other vital structures, causing 
intracranial hemorrhage and other complications $(3,16)$. In recent years, with the development of frameless image-navigation technology, the accuracy of positioning and puncturing has significantly improved, and has been applied in the implantation of epilepsy depth electrodes in some epilepsy centers, especially for SEEG, which requires the implantation of multiple depth electrodes $(1,3,13)$. Since June 2011, the Medtronic S7 neuronavigation system has been used for the implantation of brain depth electrodes at our hospital, and has achieved good results, with an average of 4.8 electrodes implanted per patient, in 36 patients with epilepsy. The method and the results are described below, and were compared with those for the previous frameless stereotactic method.

\section{MATERIAL and METHODS}

This study was conducted with approval from the Ethics Committee of the Third Military Medical University and written informed consent was obtained from all participants.

\section{General Information}

Thirty-six patients (image group), including 21 males and 15 females, aged 6-41 years, with a mean age of 18.1 years, who were implanted with depth electrodes using the imaging navigation method were assessed. The disease duration was 1-21 years, (average: 7.8 years). The seizure types observed included 8 cases of simple partial seizures, 11 cases of complex partial seizures, and 17 cases of partial seizures followed by secondary general seizures. The patients all suffered from medication-intractable epilepsy (i.e. ineffective treatment by at least two kinds of first-line antiepileptic drugs, with seizures that were frequent and seriously impacted the patients' work and lives).

The indications that intracranial electrode implantation is necessary were: 1) The patient suffered medication-intractable epilepsy; 2) The preoperative non-invasive evaluation could not clearly locate the focus position; 3) The results of non-invasive assessments, such as such as imaging, electrophysiology and symptomatology were conflicting with each other; 4) The epileptogenic foci were located close to functional areas, meaning the distinction between the two areas needed accurate determination.

Another 28 patients, who were implanted with depth electrodes (in 2009-2011) using the frame-based stereotactic method were set as the control group (the frame group). There was no significant difference in the age, sex, onset age and intelligence between the two groups (Table I), and patients who underwent a craniotomy for the grid-like electrode monitoring were excluded. This study was conducted in accordance with the declaration of Helsinki.

\section{Noninvasive Assessment Detection}

All patients were assessed for the symptomatology of onset, as well as the preoperative monitoring of active EEG (AEEG) and video EEG (VEEG) (partial patients were tested with dense electrodes, sphenoid electrodes, orbital electrodes, and induction tests). All patients were given an magnetic resonance imaging (MRI) examination, and partial patients were simultaneously given a computed tomography (CT)

Table I: Assessment Results of the 2 Groups

\begin{tabular}{|c|c|c|c|}
\hline & & Image group & Frame group \\
\hline \multicolumn{2}{|l|}{ Average age } & $18.1 \pm 6.5$ & $24.7 \pm 7.4$ \\
\hline \multicolumn{2}{|l|}{ Gender ratio (male/female) } & 1.40 & 1.55 \\
\hline \multicolumn{2}{|l|}{ Disease duration (years) } & $7.1 \pm 2.6$ & $6.3 \pm 2.7$ \\
\hline \multicolumn{2}{|l|}{ Intelligence (IQ) } & $85.9 \pm 10.4$ & $89.2 \pm 11.3$ \\
\hline \multicolumn{2}{|c|}{ The onset semiology had the positioning meaning } & $75.0 \%(27 / 36)$ & $71.4 \%(20 / 28)$ \\
\hline \multicolumn{2}{|c|}{ MRI exhibited the abnormal lesions } & $66.7 \%(24 / 36)$ & $82.1 \%(23 / 28)$ \\
\hline \multicolumn{2}{|c|}{ Scalp EEG could verify the side } & $58.3 \%(21 / 36)$ & $35.7 \%(10 / 28)$ \\
\hline \multicolumn{2}{|l|}{ PET low metabolic lesions } & $14 / 18$ & $7 / 15$ \\
\hline \multicolumn{2}{|c|}{ SPECT during the onset intervals } & $4 / 5$ & $7 / 10$ \\
\hline \multicolumn{2}{|c|}{ SPECT during the onset intervals } & $2 / 4$ & $4 / 6$ \\
\hline \multicolumn{2}{|l|}{ MRS metabolic changes } & $15 / 23$ & $8 / 16$ \\
\hline \multirow{4}{*}{$\begin{array}{l}\text { Preliminary assessment } \\
\text { revealed the involved lobes }\end{array}$} & One lobe & $50.0 \%(18 / 36)^{*}$ & $14.3 \%(4 / 28)$ \\
\hline & 2 lobes & $30.6 \%(11 / 36){ }^{*}$ & $82.1 \%(23 / 28)$ \\
\hline & 3 lobes & $11.1 \%(4 / 36)$ & $3.6 \%(1 / 28)$ \\
\hline & More than 3 & $8.3 \%(3 / 36)$ & $0.0 \%(0 / 28)$ \\
\hline
\end{tabular}

${ }^{*}$ Compared with the frame group, $p<0.05$. 
scan. The magnetic resonance spectroscopy (MRS), positron emission tomography (PET), single photon emission computed tomography (SPECT) of onset interval period and onset period, as well as the blood oxygenation level-dependent functional MRI (BOLD-fMRI) were performed according to the prophase examination results, and all the patients were subject to an intelligence assessment.

\section{Implantation Method of the Electrodes}

The image group: the MRI thin layer scanning $(1.25 \mathrm{~mm})$ was performed preoperatively, with the contrast agent bolus used to form the arterial angiography and 3D MR venography. The imaging data were transferred to the Medtronic S7 neuronavigation system (Medtronic, S7 type, Minneapolis, USA) to reconstruct 3D models of the skull, brain, and blood vessels (Figure 1A, B). These models were then combined to display the positional relationships between the blood vessels, cerebral cortex, and scalp. This was used to designate a puncturing path, including the target point and cranial-incising point (avoiding blood vessels and the major functional areas) (Figure 1C). After general anesthesia, a C-type head frame was used to fix the patient's head. With the navigation reference frame fixed in the $\mathrm{C}$-type head frame, the navigation program was registered (Figure $2 \mathrm{~A}$ ), and entered the navigation mode. The selected landmarks were used to verify navigation accuracy. Under navigation guidance, the corresponding preoperative-designed cranial-incising point was found, then combined with the Navigus tool to determine the direction of electrode implantation path (Figure 2B). A small incision was made on the skin (about $3 \mathrm{~mm}$ ). A 2.5 $\mathrm{mm}$ mini-drill was used to drill along the path direction, and electrocoagulation was performed to puncture the dura mater.
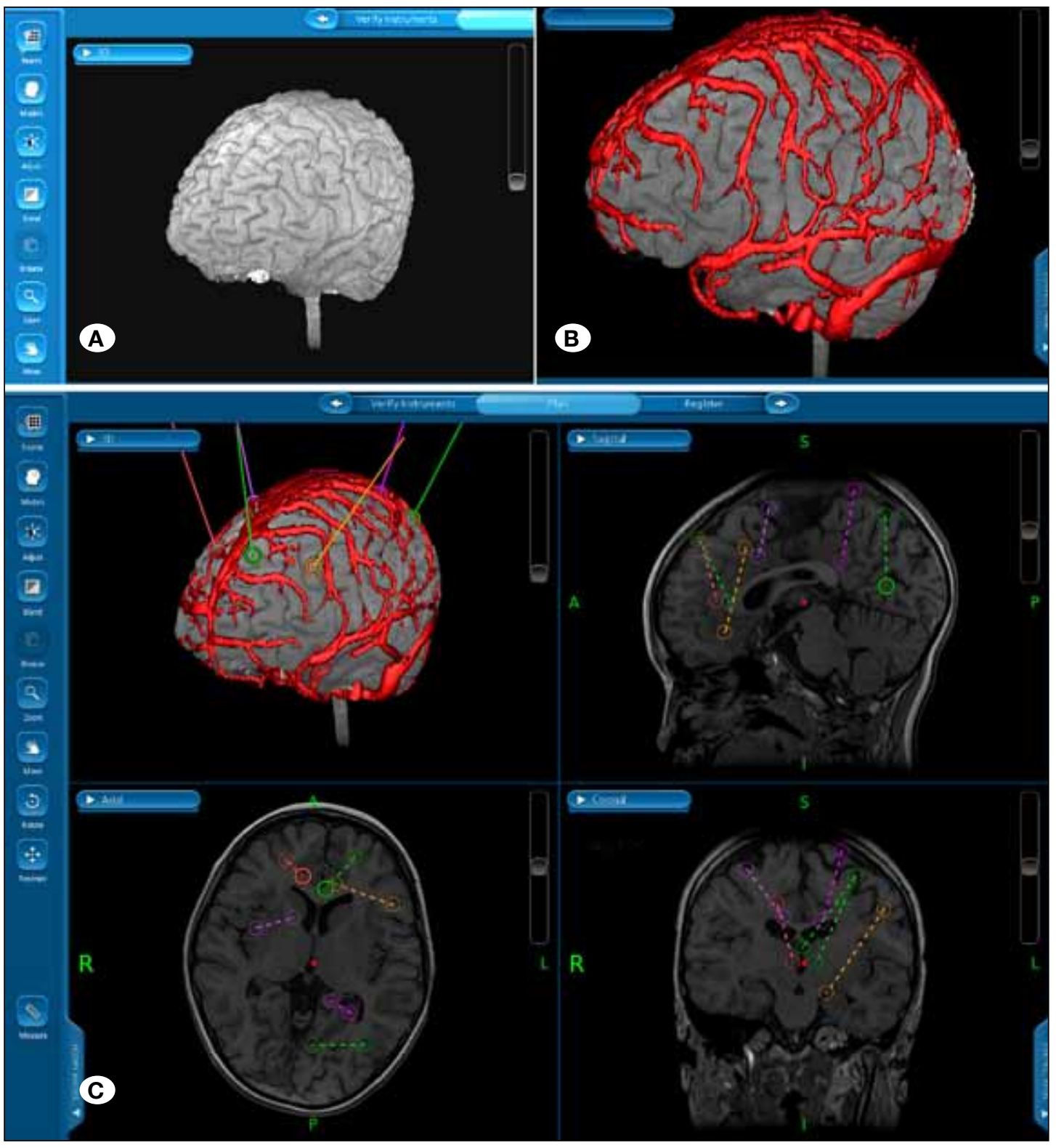

Figure 1:

Preoperative preparation of neuronavigation.

A) Reconstruction of brain;

B) reconstruction of superficial veins of brain;

C) design of puncturing path (avoiding brain vessels and major functional areas). 
The depth electrode (electrode diameter: $1.8 \mathrm{~mm}$ ) was then guided by the dedicated catheter (the catheter could instantly communicate with the navigation and display the implantation position in real-time) to reach the implantation target point. The electrode was then fixed to the skin with sutures (Figure 2C).

Implantation of strip-like electrodes: the image guidance was used to determine the skull-incising point while simultaneously observing whether there were large and dense vessels that would obstruct the electrode implantation. Subsequently, conventional drilling was performed, and 8 conductive poles implanted by hand. The frame group used the Leksell framebased stereotactic system according to the traditional method (Elekta Instrument AB, G-type Stockholm, Sweden).

\section{Post-Processing of the Electrode Implantation}

After implantation, CT scanning was performed immediately, and the fusion was reconstructed using the navigation software (Figure 2D). This was used for comparison with the $\mathrm{MRI}$ results before implantation. The predetermined electrode target position and the actual center position of the electrode were calculated to compare accuracy, which was determined using the distance between the center electrode position and the predetermined target point. Intracranial EEG monitoring was performed on the $2^{\text {nd }}$ day, and after the $3^{\text {rd }}$ day, the patient underwent an electrical stimulation test. After the operation, a pressure dressing was placed on all patients and antibiotics started from the $1^{\text {st }}$ postoperative day. After completing the intracranial EEG monitoring, the electrodes were unplugged in the dressing room.

\section{Postoperative Follow-Up and Statistical Analysis}

Anti-epileptic drugs were postoperatively administrated and adjusted according to the monitored clinical symptoms and blood concentration. The postoperative follow-up was performed once every 3-6 months, and the results of seizure control were divided according to the improved Engel classification method into the following groups: fully controlled; rare seizures; significant reduction of the postoperative seizures (the reduction rate was greater than $75 \%$ ); insignificant improvement (the reduction was less than $75 \%$ ). At the same time, EEG and neuropsychological follow-ups were also performed. Statistical analyses were carried out using SPSS18 software, and the counting data were tested with the chisquare test and Fisher's exact test. The classification data was assessed using the rank sum test, and the measurement data were assessed using the $t$-test.

\section{RESULTS}

\section{Noninvasive Assessment}

The results of MRI thin layer scanning of the 36 patients in the image group showed that 22 patients exhibited structural abnormalities, including 10 cases of hippocampal sclerosis (of which 2 cases were bilateral hippocampal sclerosis), 6 of
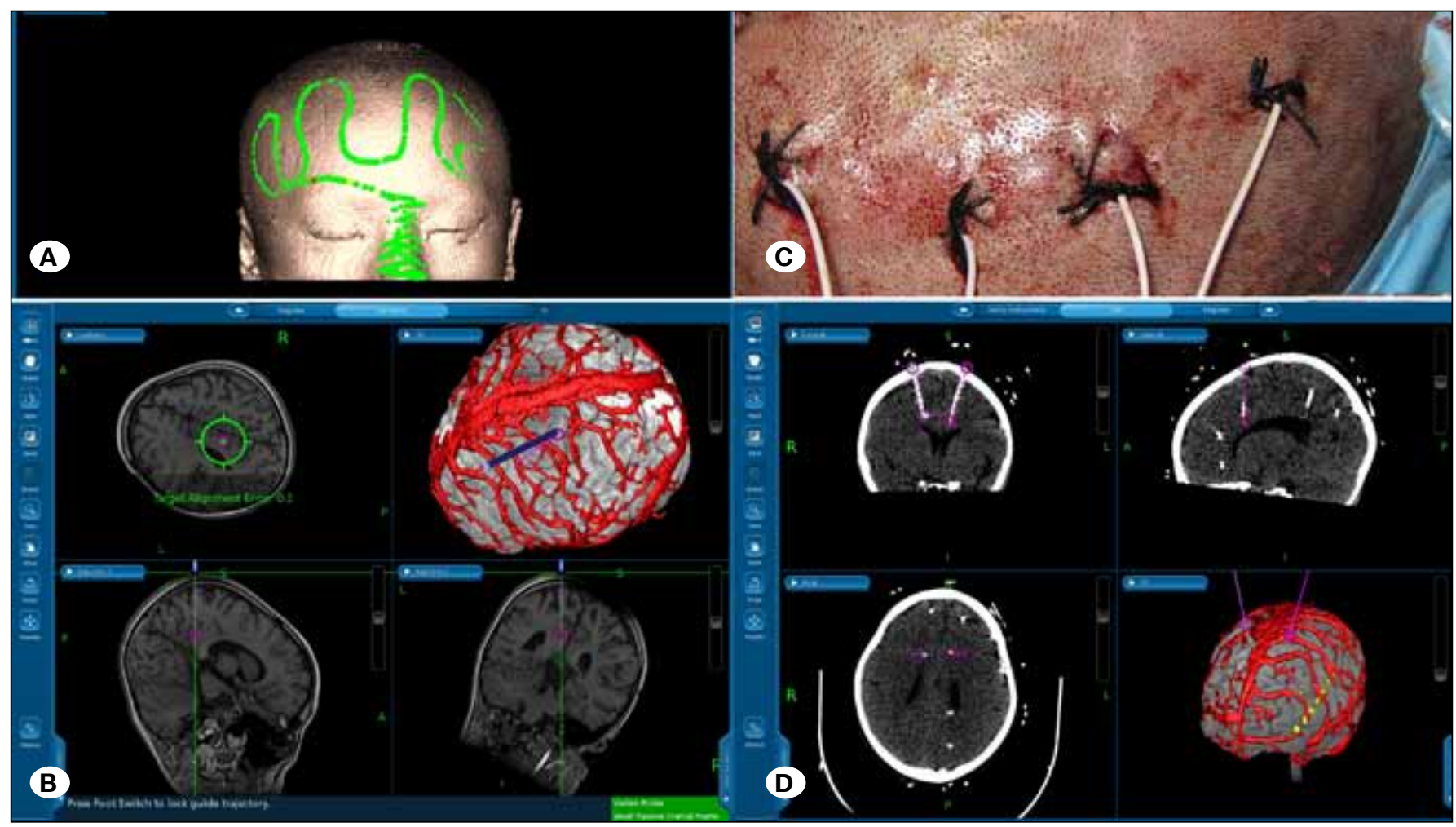

Figure 2: Intraoperative neuronavigation and postoperative image reconstruction. A) Neuronavigation program registration; B) intraoperative puncturing with neuronavigation guiding; C) fixation of electrode; D) postoperative CT scanning and image reconstruction to determine the electrode position and compare with preoperative predetermined target. 
cortical developmental disorders, 8 of brain atrophy lesions, and 6 of abnormal signal, among which 6 exhibited multiple abnormal lesions. MRI analysis ( 23 cases) indicated 15 cases of reduced temporal lobe metabolism on one side, while 8 cases were normal. PET (18 cases) showed that 14 cases exhibited low metabolic lesions. SPECT ( 5 cases) showed that 4 cases exhibited hypoperfused lesions during the seizure intervals, of which 2 showed one or more high-perfused areas during the seizures (compared with the seizure intervals). According to semiology, structural and functional imaging detections, and scalp EEG testing, the possible epileptogenic regions were preliminarily assessed. It was found that 18 cases might involve a single cerebral lobe, 11 might involve two cerebral lobes, 4 might involve 3 cerebral lobes, and 3 might involve more than 3 cerebral lobes. The preliminary assessment revealed that the involved cerebral lobes could include the temporal (25 cases), frontal (18 cases), parietal (9 cases), and occipital lobes (6 cases). The results of the frame group are shown in Table I. Compared with the image group, fewer involved cerebral lobes were identified during the preliminary assessment $(p<0.05)$.

\section{Electrode Implantation}

Thirty-six patients underwent intracranial depth electrode implantation under image navigation, with the number of depth electrodes ranging from 2-14, with a total number of 173 , and an average number of 4.8/patient. In 22 cases, 3 or more electrodes were implanted. In 14 cases, 5 or more electrodes were implanted. In 5 cases, 10 or more electrodes were implanted. As for the locations of depth electrodes, 54 were in the temporal lobe, 83 were in the frontal lobe, 20 were in the parietal lobe, and 16 were in the occipital lobe. All the depth electrodes reached the predetermined the target areas and near areas. The distance between the predetermined target area and the CT scanning result was used to calculate the accuracy: 140 electrodes were within $2 \mathrm{~mm}, 29$ electrodes were in the range of 2-4 $\mathrm{mm}$, and 4 electrodes were more than $4 \mathrm{~mm}$ away. The average distance of the electrodes from the predetermined target area was $2.03 \pm 0.98 \mathrm{~mm}$. The mean operation time for each implantation was $19.4 \mathrm{~min}$. During the implantation of depth electrodes, 24 patients from the image group were implanted with cortical strip electrodes simultaneously, with a total strip electrode count of 68 .

In the frame group, 62 depth electrodes were implanted, with an average of 2.2/patient. Forty-four electrodes were placed in the temporal lobe, 10 in the frontal lobe, 5 in the parietal lobe, and 3 in the occipital lobe. As for the implantation accuracy, 46 electrodes were within $2 \mathrm{~mm}$ of the predetermined target area, 14 were in the range of $2-4 \mathrm{~mm}$, 1 was greater than $4 \mathrm{~mm}$, the average was $1.79 \pm 0.81 \mathrm{~mm}$; the precision was better than that in the image group, but there was no significant difference $(p>0.05)$. The mean operation time for each electrode was $34.5 \mathrm{~min}$; significantly greater than that required using image guidance $(p<0.05)$. A total of 36 cortical strip electrodes were implanted in the frame group.

The electrode implantation accuracies in different locations in the 2 groups are shown in (Table II). In the image group, the accuracy in the frontal lobe was the best (with $86.7 \%$ electrode implantation within $2 \mathrm{~mm}$ of the predetermined target area), whereas in the temporal lobe it was the worst, with only $70.4 \%$ within $2 \mathrm{~mm}$, exhibiting a significant difference when compared to the frontal lobe $(p<0.05)$. There was no significant difference between other groups. In the frame group, because the electrodes were mainly concentrated in the temporal lobe, there was no significant difference between the various parts. The comparison of two groups revealed that the electrodes in the temporal lobe of the frame group were better placed than those in the image group $(p<0.05)$. There was no significant difference among the remaining groups.

\section{Complications of Electrode Implantation}

After the implantation, 1 case in the image group exhibited a small amount of high density shadow near the target point, consisting of about 1-2 ml. Intraoperative exploration revealed that it was bruising without the formation of a hematoma. During the monitoring process, 1 patient developed cerebrospinal fluid leakage, which disappeared after suturing and pressure

Table II: Accuracy Comparison of Depth Electrode Implantation in the Two Groups

Image group Frame group

\begin{tabular}{|c|c|c|c|c|c|c|c|c|}
\hline & $\begin{array}{l}\text { Mean } \\
\text { accuracy } \\
(\mathrm{mm})\end{array}$ & $\begin{array}{l}\leq 2 \mathrm{~mm} \\
(\mathrm{n}, \%)\end{array}$ & $\begin{array}{c}2-4 \mathrm{~mm} \\
(\mathrm{n}, \%)\end{array}$ & $\begin{array}{l}\geq 4 \mathrm{~mm} \\
(\mathrm{n}, \%)\end{array}$ & $\begin{array}{l}\text { Mean } \\
\text { accuracy } \\
(\mathbf{m m})\end{array}$ & $\begin{array}{l}\leq 2 \mathrm{~mm} \\
(\mathrm{n}, \%)\end{array}$ & $\begin{array}{c}2-4 \mathrm{~mm} \\
(\mathrm{n}, \%)\end{array}$ & $\begin{array}{c}\geq 4 \mathrm{~mm} \\
(\mathrm{n}, \%)\end{array}$ \\
\hline Frontal lobe & $1.84 \pm 0.86^{*}$ & $72(86.7)$ & $10(12.0)$ & $1(1.2)$ & $1.72 \pm 0.81$ & $8(80.0)$ & $2(20.0)$ & 0 \\
\hline Temporal lobe & $2.31 \pm 1.09^{* *}$ & $38(70.4)$ & $14(25.9)$ & $2(3.7)$ & $1.82 \pm 0.86$ & $37(84.1)$ & $6(13.6)$ & $1(2.3)$ \\
\hline Parietal lobe & $1.93 \pm 0.72$ & $17(85.0)$ & $3(15.0)$ & 0 & $1.42 \pm 0.32$ & $4(100.0)$ & 0 & 0 \\
\hline Occipital lobe & $2.18 \pm 1.72$ & $13(78.6)$ & $2(14.3)$ & $1(7.1)$ & $2.04 \pm 0.68$ & $3(75.0)$ & $1(25.0)$ & 0 \\
\hline Summary & $2.03 \pm 0.98$ & $140(80.9)$ & 29 (16.8) & $4(2.3)$ & $1.79 \pm 0.82$ & 52 (83.9) & $9(14.5)$ & $1(1.6)$ \\
\hline
\end{tabular}

*Versus the temporal lobe of the image group, $p<0.05$.

*Versus the temporal lobe of the frame group, $p<0.05$. 
Hou Z. et al: Neuronavigation vs. Frame-Based Stereotaxy

dressing. No cerebrospinal fluid leakage was found after the removal of electrodes. The image group exhibited no intracranial infection or scalp infection. Four patients exhibited mental abnormalities during the EEG monitoring, and 1 patient selfpulled out the electrodes. While the CT scan showed no hematoma or other abnormalities, the symptoms spontaneously remitted after the electrodes were removed. The frame group exhibited 2 cases of intracranial hematoma, 3 of cerebrospinal fluid leakage, 1 of intracranial infection, and 3 of mental disorders (Table III).

\section{Intracranial Electrode Monitoring and Surgeries}

The intracranial EEG monitoring time for the image group was 3-18 days, with an average of 8.9 days. In 32 cases, the epileptogenic loci could be determined through $\mathrm{EEEG}(88.9 \%)$, with the number of epileptogenic foci being 1-2. In all cases surgical resection was performed. In the other 4 cases, the epileptogenic foci could not be determined or were called into question, in which case the surgery was not performed. The surgical resections of epileptogenic foci were all performed according iEEG monitoring results. The postoperative followups were conducted in 3-24 months, and in 21 cases the epilepsy could be fully controlled. In 5 cases, rare postoperative seizures were observed, and in 4 cases, the postoperative seizures were significantly reduced (the reduction was greater than $75 \%$ ). In 2 cases, there was no significant improvement. In the frame group, the epileptogenic foci of 23 cases could be determined accurately (82.1\%). In the remaining 5, surgery was not performed because the epileptogenic foci could not be determined. The postoperative follow-ups were conducted in 12-36 months. In 14 cases, the epilepsy could be fully controlled, in 4 cases, rare postoperative seizures were observed, in 2 cases, the postoperative seizure were significantly reduced, and in 3 cases, the patients exhibited no significant improvement. There was no difference between the 2 groups $(p<0.05)$.

\section{DISCUSSION}

With the development of epilepsy surgery, the role of invasive iEEG has become increasingly prominent. Intracranial electrodes can be classified into epidural, subdural cortical, and depth electrodes, of which depth electrodes play a very important role in the evaluation of epileptogenic focus for epilepsy surgery, for example in mesial temporal lobe epilepsy and frontal lobe epilepsy $(12,21)$. There was also a study that showed that depth electrodes exhibited better side-determining effects than did subdural strip electrodes in cortical epilepsy (21). The previous implantation of depth electrodes usually used the frame-based stereotactic method, and although it provided accurate implantation locations, the surgical procedures were much more complex. When time and tests are required, such as during a cerebral angiography, it would be difficult to implant multiple depth electrodes (9, 16). Meanwhile, the puncturing process could injure blood vessels and important functional areas, thus increasing complications such as intracranial bleeding $(2,9)$. With the development of imaging techniques, the image-navigated depth electrode implantation has become a trend in some epilepsy
Table III: Comparison of Complications in the Two Groups

\begin{tabular}{lcc}
\hline & Image group & Frame group \\
\hline Hematoma & 0 & 2 \\
\hline Infection & 0 & 1 \\
\hline Cerebrospinal fluid leakage & 1 & 3 \\
\hline Mental abnormality & 4 & 3 \\
\hline Electrode pull-out & 1 & 0 \\
\hline
\end{tabular}

centers. High-precision image navigation can achieve accuracy as good as $1 \mathrm{~mm}$, and has already been able to meet the demands of depth electrode implantation $(3,18)$. The results of this study group showed that, compared with the conventional frame-based stereotactic method, the accuracy in the image group was of a similar degree, while the operation time was reduced significantly. At the same time, complications such as bleeding and cerebrospinal fluid leakage were also significantly reduced, indicating that the image-navigated depth electrode implantation could replace the frame-based stereotactic method and become an important means for depth electrode implantation $(2,9)$.

Accuracy is key to depth electrode implantation, and the average deviation of the image group was $2.03 \mathrm{~mm}$. More than $80 \%$ of electrodes were within $2 \mathrm{~mm}$ of the predetermined position, exhibiting no significant difference when compared to the frame method. Meanwhile, the results showed that the accuracy difference of the 2 groups was only $0.24 \mathrm{~mm}$. Because the accuracy requirement in epilepsy is lower than in other diseases, this low difference indicates that neuronavigation could meet the accuracy requirement for depth electrode implantation $(11,16)$. Widmann et al. (20) summarized the literature published between 1995-2010, and found that the overall accuracy of frame stereotactic system was slightly higher than the image navigation system. The accuracy requirements of the targets should be considered, and the kind of method to be applied should be determined on a case by case basis. The frameless positioning system might exhibit much more obvious advantages when implanting depth electrodes, such as less time-consumption and simple operation, which was also proved in this study. The operation time in the frameless group (the neuronavigation group) was significantly lower than that in the frame group. With the development of neuronavigation technology, the accuracy could be constantly improved. The accuracy in this study was better than those in earlier foreign reports, and similar to those in recent reports $(2,16,18,21)$. Ortler et al. compared the results of VBH frameless positioning systems (combined with neuronavigation) and Fischer-Leibinger frame systems for depth electrode implantation, and found that they had similar accuracies (16). In this study, the new generation neuronavigation approach exhibited more simplicity than Ortler's VBH frameless positioning system, due to no need for the installation of a positioning frame, the shorter time consumption, and a simpler operation. 
Although image navigation and stereotactics can achieve a degree of accuracy of less than $1 \mathrm{~mm}$, it did not achieve such accuracy in this study, which might be related to the electrode fixation method. In this study, the electrodes were fixed through the skin. A relatively large postoperative shift might lead to positional deviation. The fixing screws widely used in some centers, which are supplied by the commercial companies, might improve the electrode accuracy $(16,18)$. Among the electrodes in different locations, the temporal lobe position significantly affected accuracy, while the frontal lobe and occipital lobe did not. This might be because the temporal lobe is covered by a distinct muscle layer that may cause a shift in electrode position.

Complications and safety are often the focus of intracranial electrode implantation $(5,7,17)$. This study successfully used the image navigation method, with only 1 case exhibiting a small amount of bleeding. The incidences of infection and cerebrospinal fluid leakage were also lower than in the frame group, indicating the superiority of image-navigated depth electrode implantation. In this study, the image navigation software could reconstruct the veins, arteries, and important functional areas, and thus could avoid them during the surgery, reducing the chance of bleeding. Meanwhile, the vascular reconstruction allowed small incisions and micro bone holes for the puncturing, reducing the incidence of cerebrospinal fluid leakage, infection, and other complications. In 41 reported cases of image-guided electrode implantation, there was no occurrence of bleeding, infection, or other serious complications (12). The case reports of the Montreal Neurological Hospital (MNI) reported that 217 patients were implanted with 3022 electrodes using the image guidance technique, and the results showed that there were only 3 cases of bleeding, and 3 cases of abscess, which further demonstrate that the intracranial electrode implantation performed with video navigation is the safer method (7).

With the development of image navigation technology and frameless stereotactic technology, close attention has been paid to stereoelectroencephalography (SEEG), performed using multiple depth electrodes as well as other cortical electrodes. This is due to the comprehensive and 3D information that can be obtained $(4,7,10)$. SEEG could not only be used to monitor the initial part of the epileptic onset clinically, but also the sites that propagated the epileptiform discharges. The performance of epileptiform discharges appear as a multi-direction 3D conception (10). In this group the image navigation method was used to implant multiple depth electrodes (5-14 electrodes in this study) into 14 patients, and combined with the cortical electrodes that were implanted at the same time, an SEEG that could monitor the initiation and propagation of epileptiform discharges was established. The results here show that SEEG exhibits the advantage of distinguishing a number of suspicious epileptogenic foci and bilateral suspicious epileptogenic foci, as well as extensive deep lesions. The neuronavigation was a better tool for the establishment of SEEG, with the advantages of ease of operation and fewer complications $(12,15,19)$.
Because epilepsy surgery was initiated late in China, subdural cortical electrodes are widely used as a means for monitoring intracranial EEG. SEEG has being applied in China in recent years, and this was the first study that used the neuronavigation to establish SEEG. Because SEEG has been carried out in China recently, when compared with other countries, the corresponding technical systems and equipment are still not optimal. Some commercial equipment are not yet available in the country, and the dedicated electrodes and fixed equipment are still lacking; therefore, the accuracy of electrodes and electrode monitoring might be affected. Further exploration and establishment of SEEG and its technical methods would be very appropriate and advantageous in developing countries (8).

\section{ACKNOWLEDGMENTS}

The research work of the authors was supported by grants from National Natural Science Foundation of China (no.81371424, and 81201011).

\section{REFERENCES}

1. Bararo NM: Stereoelectroencephalography using computerized tomography-or magnetic resonance imaging-guided electrode implantation. J Neurosurg 104(4):480-482, 2006

2. Cardinale F, Cossu M, Castana L, Casaceli G, Schiariti MP, Miserocchi A, Fuschillo D, Moscato A, Caborni C, Arnulfo G, Lo Russo G: Stereoelectroencephalography: Surgical methodology, safety, and stereotactic application accuracy in 500 procedures. Neurosurgery 72(3):353-366, 2013

3. Centeno RS, Yacubian EM, Caboclo LO, Junior HC, Cavalheiro S: Intracranial depth electrodes implantation in the era of image-guided surgery. Arq Neuropsiquiatr 69(4):693-698, 2011

4. Cossu M, Cardinale F, Castana L, Citterio A, Francione S, Tassi L, Benabid AL, Lo Russo G: Stereoelectroencephalography in the presurgical evaluation of focal epilepsy: A retrospective analysis of 215 procedures. Neurosurgery 57(4):706-718, 2005

5. Cossu M, Cardinale F, Castana L, Nobili L, Sartori I, Lo Russo G: Stereo-EEG in children. Childs Nerv Syst 22(8):766-778, 2006

6. Dalal SS, Edwards E, Kirsch HE, Barbaro NM, Knight RT, Nagarajan SS: Localization of neurosurgically implanted electrodes via photograph-MRI-radiograph coregistration. J Neurosci Methods 174(1):106-115, 2008

7. De Almeida AN, Olivier A, Quesney F, Dubeau F, Savard G, Andermann F: Efficacy of and morbidity associated with stereoelectroencephalography using computerized tomography or magnetic resonance imaging-guided electrode implantation. J Neurosurg 104(4):483-487, 2006

8. Gonzalez-Martinez J, Mullin J, Vadera S, Bulacio J, Hughes G, Jones S, Enatsu R, Najm I: Stereotactic placement of depth electrodes in medically intractable epilepsy. J Neurosurg 120(3):639-644, 2014 
9. Guenot M, Isnard J, Ryvlin P, Fischer C, Ostrowsky K, Mauguiere F, Sindou M: Neurophysiological monitoring for epilepsy surgery: The Talairach SEEG method. Stereo Electro Encephalo Graphy. Indications, results, complications and therapeutic applications in a series of 100 consecutive cases. Stereotact Funct Neurosurg 77(1-4):29-32, 2001

10. Kahane P, Landre E, Minotti L, Francione S, Ryvlin P: The Bancaud and Talairach view on the epileptogenic zone: A working hypothesis. Epileptic Disord 8:S16-S26, 2006

11. McGonigal A, Bartolomei F, Regis J, Guye M, Gavaret M, Trébuchon-DaFonsecaA, Dufour H, Figarella-BrangerD, Girard $\mathrm{N}$, Péragut JC, Chauvel P: Stereoelectroencephalography in presurgical assessment of MRI-negative epilepsy. Brain 130(Pt 12):3169-3183, 2007

12. McGonigal A, Gavaret M, Da Fonseca AT, Guye M, Scavarda $\mathrm{D}$, Villeneuve $\mathrm{N}$, Régis J, Bartolomei $\mathrm{F}$, Chauvel P: MRInegative prefrontal epilepsy due to cortical dysplasia explored by stereoelectroencephalography (SEEG). Epileptic Disord 10(4):330-338, 2008

13. Mehta AD, Labar D, Dean A, Harden C, Hosain S, Pak J, Marks D, Schwartz TH: Frameless stereotactic placement of depth electrodes in epilepsy surgery. J Neurosurg 102(6):1040-1045, 2005

14. Munyon CN, Koubeissi MZ, Syed TU, Lüders HO, Miller JP: Accuracy of frame-based stereotactic depth electrode implantation during craniotomy for subdural grid placement. Stereotact Funct Neurosurg 91(6):399-403, 2013

15. Murphy MA, O'Brien TJ, Cook MJ: Insertion of depth electrodes with or without subdural grids using frameless stereotactic guidance systems-technique and outcome. $\mathrm{Br} \mathrm{J}$ Neurosurg 16:119-125, 2002
16. Ortler M, Sohm F, Eisner W, Bauer R, Dobesberger J, Trinka E, Widmann G, Bale R: Frame-based vs frameless placement of intrahippocampal depth electrodes in patients with refractory epilepsy: A comparative in vivo (application) study. Neurosurgery 68(4):881-887, 2011

17. Robles SG, Gelisse P, El Fertit H, Tancu C, Duffau H, Crespel A, Coubes P: Parasagittal transinsular electrodes for stereoEEG in temporal and insular lobe epilepsies. Stereotact Funct Neurosurg 87(6):368-378, 2009

18. Serra C, Huppertz HJ, Kockro RA, Grunwald T, Bozinov O, Krayenbühl N, Bernays RL: Rapid and accurate anatomical localization of implanted subdural electrodes in a virtual reality environment. J Neurol Surg A Cent Eur Neurosurg 74(3):175182,2013

19. Vadera S, Mullin J, Bulacio J, Najm I, Bingaman W, GonzalezMartinez J: Stereoelectroencephalography following subdural grid placement for difficult to localize epilepsy. Neurosurgery 72(5):723-729, 2013

20. Widmann G, Schullian P, Ortler M, Bale R: Frameless stereotactic targeting devices: Technical features, targeting errors and clinical results. Int J Med Robot 8(1):1-16, 2012

21. Wray CD, Kraemer DL, Yang T, Poliachik SL, Ko AL, Poliakov A, Hebb AO, Novotny EJ, Ojemann JG: Reehand placement of depth electrodes using electromagnetic frameless stereotactic guidance. J Neurosurg Pediatr 8(5):464-467, 2011 